\title{
Microelectronic Engineering
}

\section{Capacitive effects in pinpin photodiodes}

\author{
A. Fantoni ${ }^{a, b, *}$, M. Fernandes ${ }^{a, b}$, P. Louro ${ }^{a, b}$, M.A. Vieira ${ }^{a, b}$, M. Vieira ${ }^{a, b, c}$ \\ aSEL-ADEETC, R. Conselheiro Emidio Novarto, 1949-014 Lisboo, Portugal \\ 'CIS-UNINOVA, Quinto da Torre, Monte da Caparica, 2829-516 Caparica, Portugal \\ ' DEE-FCT-UNL, Quinta da Torte, Monte da Caparica, 2829-516 Caparica, Portugal
}

\section{A R TICLE I N F O}

\section{Article history:}

Available online 31 January 2013

\section{Keywords}

Amorphous silicon

Photodiode

Photocapacitance

\begin{abstract}
A B S T R AC T
The application of a-SiC:H/a-Si:H pinpin photodiodes for optoelectronic applications as a WDM demultiplexer device has been demonstrated useful in optical communications that use the WDM technique to encode multiple signals in the visible light range. This is required in short range optical communicarion applications, where for costs reasons the link is provided by Plastic Optical Fibers. Characterization of these devices has shown the presence of large photocapacitive effects. By superimposing background illumination to the pulsed channel the device behaves as a filter, producing signal attenuation, or as an amplifier, producing signal gain, depending on the channel/background wavelength combination.

We present here results, obtained by numerical simulations, about the internal electric configuration of a-SiC:H/a-Si:H pinpin photodiode. These results address the explanation of the device functioning in the frequency domain to a wavelength tunable photo-capacitance due to the accumulatlon of space charge localized at the botrom diode that, according to the Shockley-Read-Hall model, it is mainly due to defect trapping. Experimental result about measurement of the photodiode capacitance under different conditions of illumination and applied bias will be also presented. The combination of these analyses permits the description of a wavelength controljed photo-capacitance that cornbined with the series and parallel resistance of the diodes may result in the explicit definition of cut off frequencies for frequency capacitive filters activated by the light background or an oscillatory resonance of photogenerated carriers between the two diodes.
\end{abstract}

2013 Elsevier B.V. All rights teserved.

\section{Introduction}

Amorphous silicon, with an optical gap of about $1.75 \mathrm{eV}$, finds its spectral photosensitivity centred in the visible spectrum, in a window ranging from red to violet wavelengths. The optical gap of a-Si:H can be enlarged by the introduction of small quantities of carbon during the deposition process. The optical gap of a-Si $\mathrm{S}_{1}-x$ $\mathrm{C}_{x}: \mathrm{H}$ can be increased up to $2.5 \mathrm{eV}$, reducing light absorption in the red part of the spectrum. Anyway, the introduction of carbon also determines a worsening of the transport properties of the material; that is, a increased density of localized states and a lower carrier mobility, resulting in a lower electrical conductivity. The physics of a-Si:H p-i-n structures for solar cells applications has been largely studied during the 1990 s. Efficiency and stability of a-Si:H solar cells has been increased up to a reasonable commercial level by developing structures based on tandem configuration and incorporating a-SiC;H layers on the top of the device. Developed

\footnotetext{
* Corresponding author at: ISEL-ADEETC, R. Conselheiro Enídio Navarro, 1949014 Lisboa, Portugal, Tel.: +351 218317289 ; Pax: +351218 317114

E-mail address: afantonifdetc.iselipl.pt (A Fantoni).
}

as photovoltaic devices a-Si:H structures have been mainly studied and characterized under steady state conditions, directing the transient measurement to the development of characterization techniques for determination of material properties. At the same time, photodiodes based on crystalline materials have been largely used as first choice receiver in optical communication system due to the high sensitivity to the infrared radiation. The good IR optical and transport properties of erystalline silicon has boosted the development of these device for communication systems with a very high transmission rate. Amorphous silicon devices were not considered for these applications for two main reasons: the attenuation characteristics of the silica optical fibres in the visible range and the slow response time due to the low mobility of the amorphous semiconductor.

The need of reducing costs of digital home appliances and car networks has recently turned on the interest in systems based on Plastic Optical Fibers (POFs) interconnections $[1,2]$. In the authors knowledge, no commercial demux system for visible range WDM-POF transmission is presently available. As the transmission window of POF is centred in the visible range and being the main interest focused in reducing the production costs, more than in 
high speed transmission rate, a-Si devices become an appealing choice within this context. Amorphous silicon photodiodes can be therefore considered as promising candidates for applications in visible range short distance communication systems. Photodiodes based on a-Si:H/a-SiC:H multilayer structure have been recently demonstrated to be capable of working as a integrared receiver/demux device in Wavelength Division Multiplexing (WDM) optical transmission systems in the visible range [3]. The device is composed of two stacked $p-i-n$ photodiodes, both optimized for the selective collection of photo generated carriers. Band gap engineering was used to adjust the photogeneration and recombination rates profiles of the intrinsic absorber regions of each photodiode to short and long wavelength absorption and carrier collection in the visible spectrum. The demux process is based on analyzing and comparing the photocurrent obtained under forward and reverse bias [3]. The produced photocurrent is used as an input for a demux algorithm based on the voltage controlled sensitivity of the device [4]. Such device has been measured and characterized under condition of a relatively low rate of transmission (up to few $\mathrm{kHz}$ ). Considering the typical POF maximum bandwidth (100 Mbit $/ 100 \mathrm{~m}$ ), transmission rates in the $\mathrm{MHz}$ regime (for a single channel) should be obtained in order to produce an improvement suitable of a real world application. We point otut the device capacitance as a main factor of limitation for transmission rate improvement. It is therefore needed a better understanding of the role played by the interfaces and the bulk intrinsic layers on the formation of the device capacitance. Preliminary results address the explanation of the device functioning in the frequency domain to a wavelength tuneable photo-capacitance due to the accumulation of space charge localized at the internal junctions [5]. This paper presents results and discussion about the observed capacitive effects.

\section{Device configuration}

The device under study was produced by PECVD (Plasma Enhanced Chemical Vapour Deposition) and optimized for a proper fine tuning of a specific wavelength. The device structure consists of a $\mathrm{p}^{\prime} \mathrm{i}^{\prime}(\mathrm{a}-\mathrm{SiC}: \mathrm{H})-\mathrm{n} / \mathrm{p}-\mathrm{i}(\mathrm{a}-\mathrm{Si}: \mathrm{H})-\mathrm{n}$ heterostructure with low conductivity a-SiC:H doped layers. Device configuration is depicted in Fig. 1 together with a picture of the real device used for the measurements. The active area of the photodiode is about $1 \mathrm{~cm}^{2}$. The thicknesses and optical gap of the thin $i^{\prime}-(200 \mathrm{~nm} ; 2.1 \mathrm{eV})$ and thick $\mathrm{j}-(1000 \mathrm{~nm} ; 1.8 \mathrm{eV})$ layers are optimized for light absorption in the blue and red ranges, respectively [6]. As a result, both front and back pin structures act as optical filters confining, respectively, the blue and the red optical carriers. By providing a complex illumination of the device combining radiations with different and complementary penetration depths it is possible to control the spectral response of the device, Roughly speaking, when the device is illuminated by red light, its spectral sensitivity is enhanced in the blue region, while the application of a blue optical bias boosts the response in the red region.

\section{Experimental results}

In Fig. 2a is reported the spectral response of the device. The measurement is performed by shining on the top surface a pulsed monochromatic light under reverse bias $(-8 \mathrm{~V})$ and the plot reports the collected photocurrent. The device presents a good sensitivity over the entire visible spectrum. It is possible to observe that the spectral response comes from the added contribution of the two diodes, being the top one responsible for the absorption in the blue region, while the bottom diode absorbs the radiation in the red part of the spectrum. The measurements have been
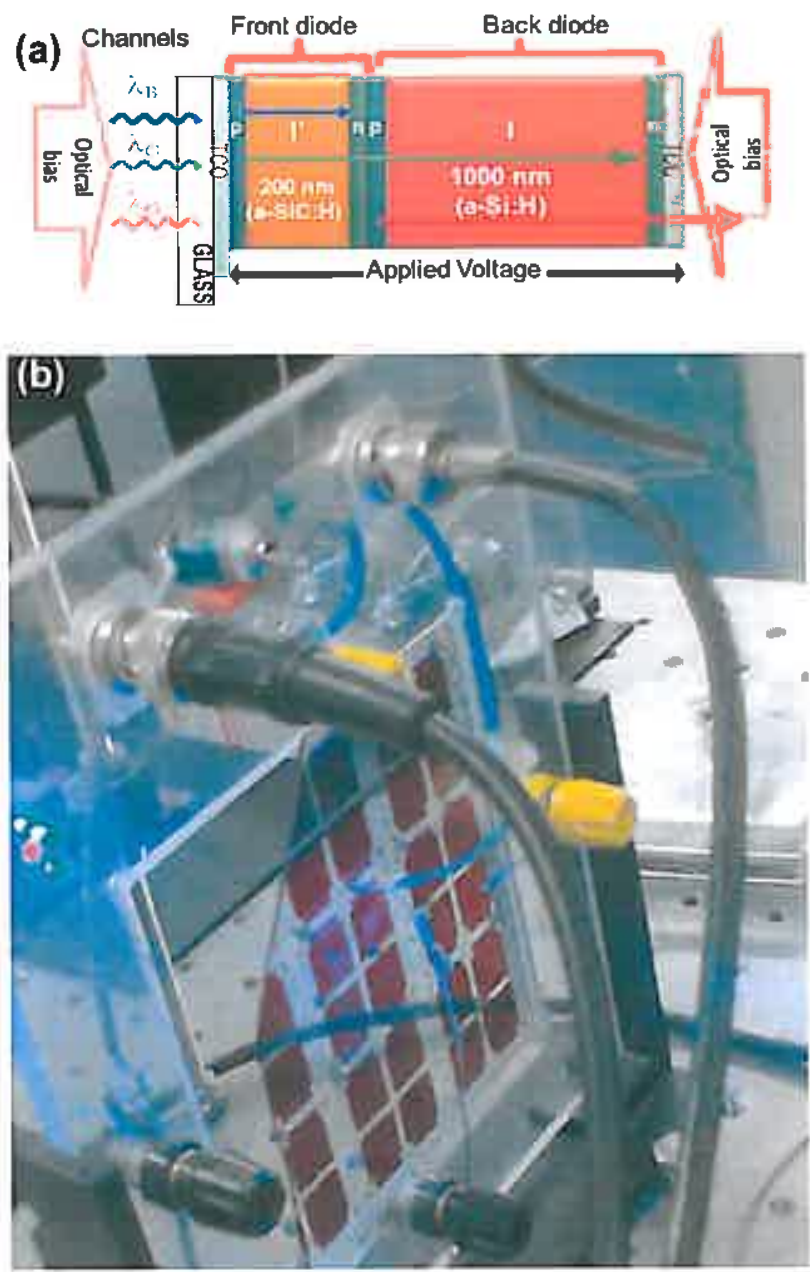

Flg. 1. Device configuration and a picture of the real device used for the measurements. The thicknesses and optical gap of the thin i' $-(200 \mathrm{~nm} ; 2.1 \mathrm{eV}$ ) and thıck $\mathrm{i}-(1000 \mathrm{~nm} ; 1.8 \mathrm{eV})$ layers are optimized for light absorption in the blue and red ranges, respectively. The active area of the photodiode is about $1 \mathrm{~cm}^{2}$. (For interpretation of the references to color in this figure legend, the reader is referred to the web version of this article.)

performed with an increasing value of the frequency of the pulsed light up to $3.5 \mathrm{kHz}$. It is possible to observe in Fig. $2 \mathrm{~b}$ a progressive decreasing of the output photocurrent with the increasing frequency. This behaviour can be ascribed to the low value of the carrier mobility and the presence of localized states where the photogenerated charges fall trapped, Under forward bias conditions the spectral response is reduced for wavelengths lower that $550 \mathrm{~nm}$. The behaviour with the increasing frequency maintains the same profile.

The application of an optical bias enhances the collection in the complementary wavelength. In Fig. 3 is plotted the ratio between the photocurrents measured with and without the application of optical bias of three wavelengths: 450,550 , and $650 \mathrm{~nm}$. It can be observed that under red and blue optical bias, independently of the frequency of the pulsed light, the device produce a gain of about $10 \mathrm{~dB}$ for the complementary wavelength. The application of a green optical bias does not produce any selection based on the wavelength of the pulsed light, but presents a strong dependence on the frequency. The device behaves as a high pass filter with a cut off frequency of about $2.5 \mathrm{kHz}$. Analyzing the response (rising and decaying) time for different wavelengths with and 

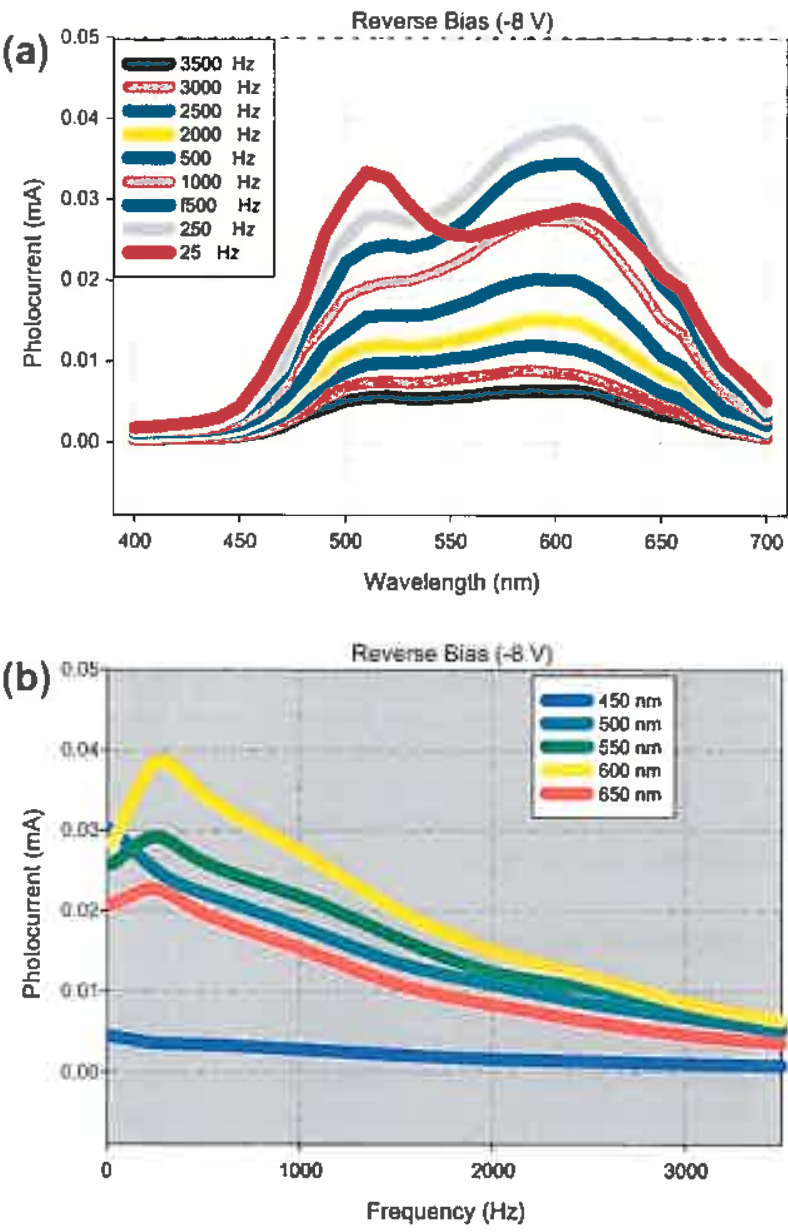

Fig. 2. Spectral response of the devire under reverse bjas $(-8 \mathrm{~V})$ : (a) Photocurrent versus wavelength for different frequencies of the pulsed light. (b) Photocurrent versus frequencies of the pulsed light lor diflerent wavelengths.

without optical bias we may conclude that the presence of the optical bias controls selectively the intensity of the current produced by the device. The delay time of the device response has no strong dependence on the optical bias.

Capacitance measurements revealed that the incidence of a light, with wavelength in the visible range, produces an increasing capacitance value whose value depends directly on the light intensity. The application of a reverse bias reduces the value of the capacitance induced by the light only in the green/blue range. In Fig. 4 it is reported the value of the device capacitance with optical bias and without. The optical bias is produced illuminating the device with LED lights. Light intensity is normalized by regulating the value of the current driving the LED. The value of the light induced capacitance depends on external electrical bias (slightly), light intensity, light wavelength. It may be observed that the light induced capacitance increases more when the device is illuminated by red light, that is, when the bottom diode is light activated, while the bottom one remains in dark conditions. The measured capacitance depends also on the experimental condirion; mainly from the frequency regime chosen for the AC modulation. All the measurement presented in this paper are obtained with a AC modulation of $1 \mathrm{kHz}$, a value of the same order of magnitude of the pulsed light used for the optical signal modulation. Increasing the frequency of the AC modulation, under reverse bias, the device capacitance suffers a light lowering. probably due to the lifetime of the charge trapped into the deep defect localized states.

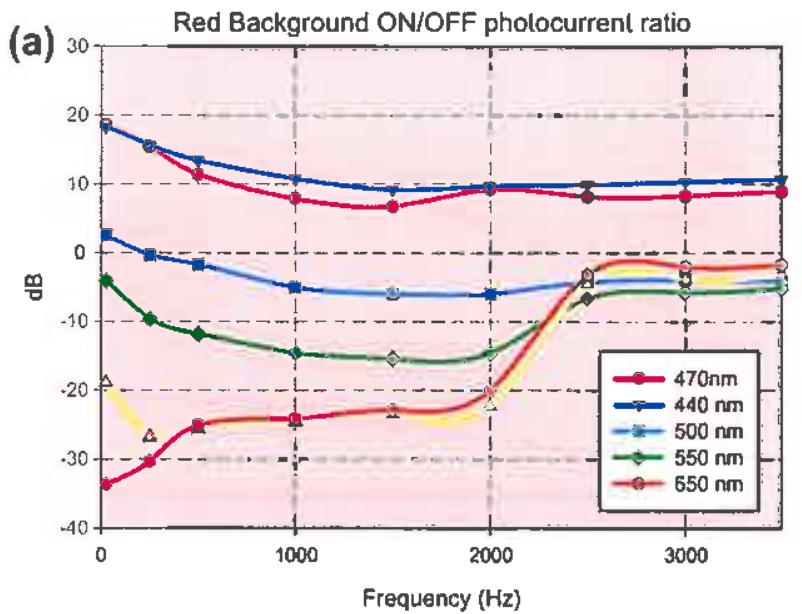

Blue Background ON/OFF pholocurrent ralio
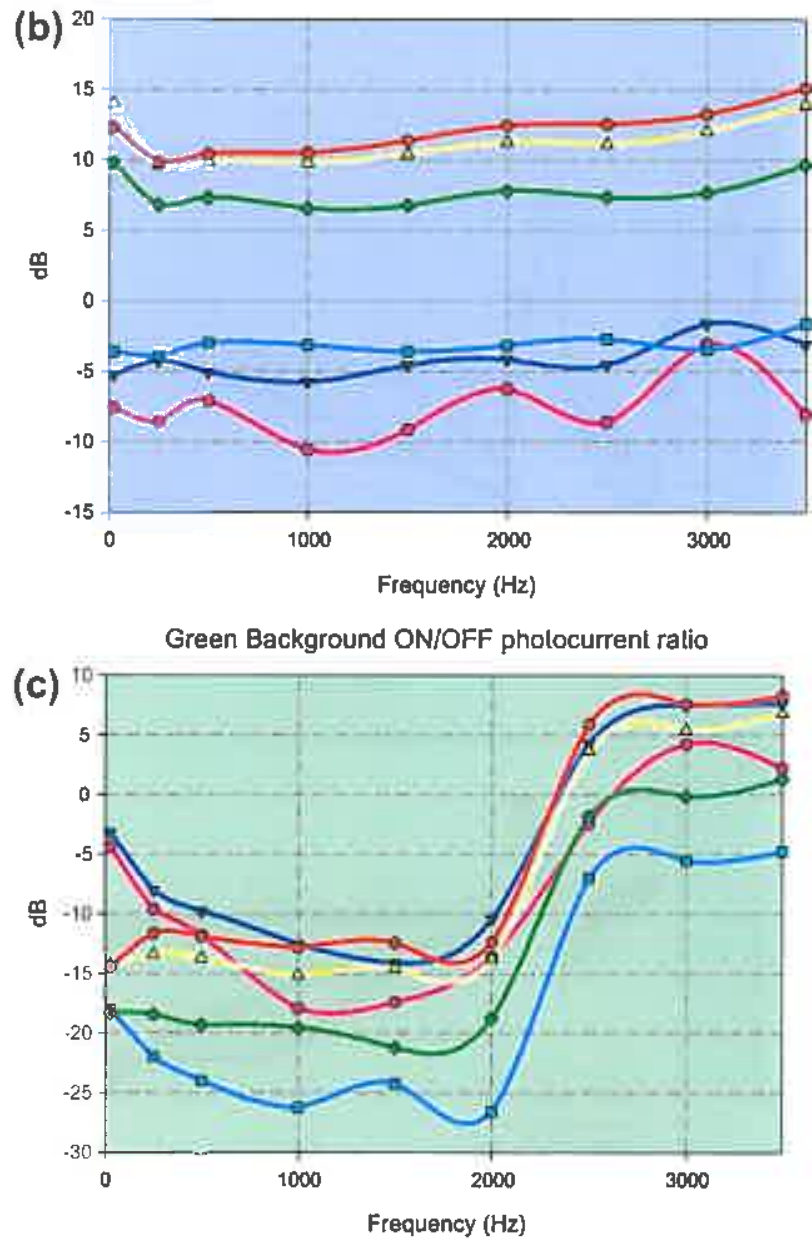

Fig- 3. Photo-bias and frequency: signal ratio between the photocurrent measured with optical bias and without optical bias. (a) Optical bias $650 \mathrm{~nm}$ (b) optical bias $550 \mathrm{~nm}$, and (c) optical bias $450 \mathrm{~nm}$.

\section{Simulation results}

The simulation program ASCA2D $[7,8]$ has been used to simulate the electrical properties of the a-Si;H/a-SiC:H pinpin device. The simulation is aimed to individuate, under different jllumination 


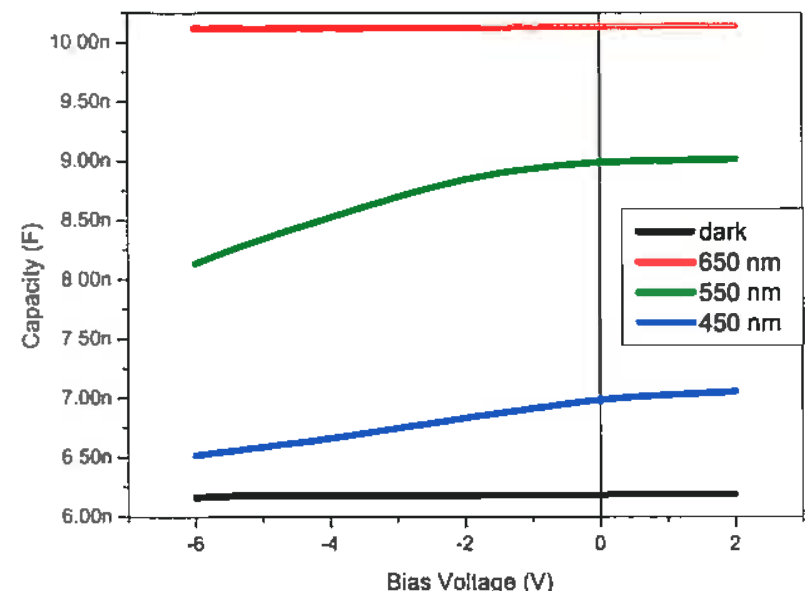

Fig. 4. Capacitance of the pinpin photodiorle measused under dark conditions and when illuminated with light of different wavelengths. Active area ot the photodiode is $1 \mathrm{~cm}^{2}$.
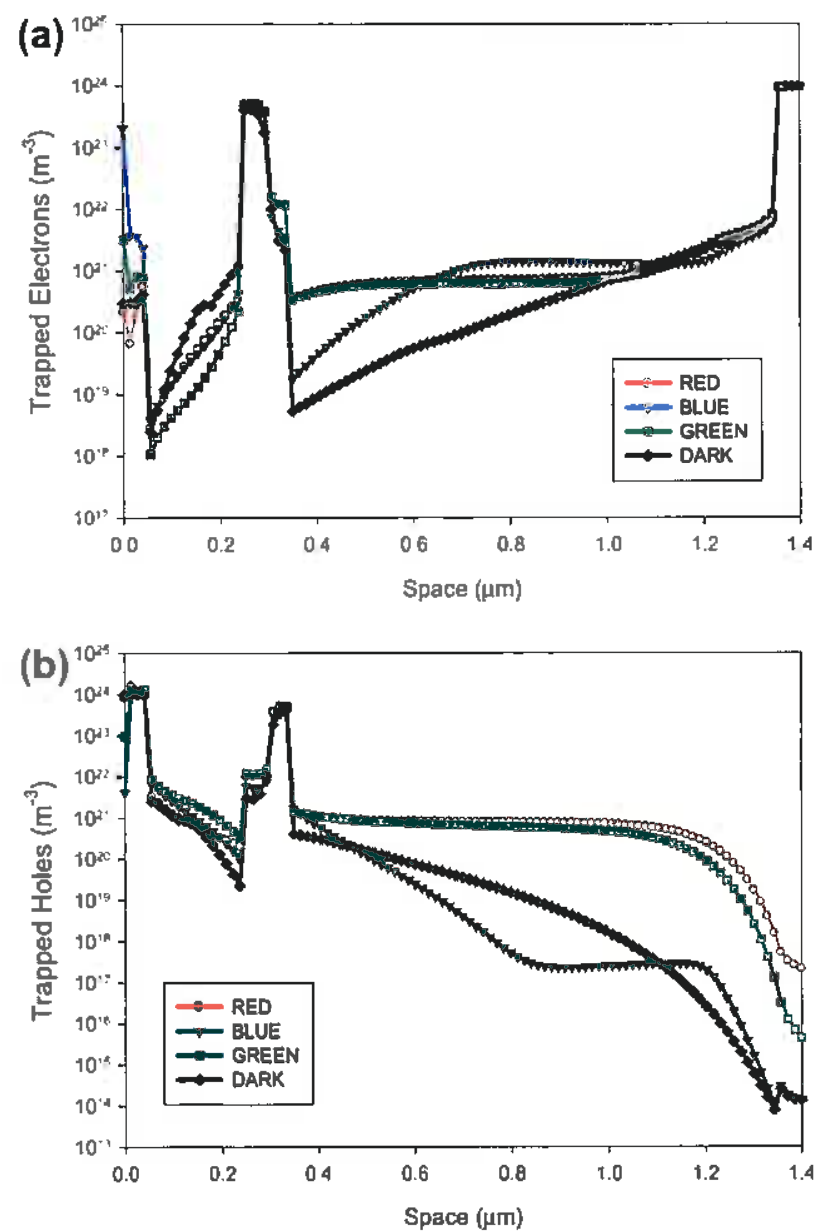

Fig. 5. Simulation results about the distribution of the trapped electrons (a) and holes (b) within the pinpin photodiode illuminated with different wavelengths.

conditions, the regions where electric charge accumulates. This information is crucial in order to understand, control and eventually exploit the phoro-capacitance effect. It must be remarked that the ASCA simulator calculates the space charge distribution using the Shockley-Read-Hall model, which is the most suitable for simulation of amorphous semiconductors. Following this model the space charge distribution is mainly due to the charge trapped into the defects states caused by the disordered structure of a-Si:H. Fig. 5 reports the distribution of the trapped electrons and holes under different illumination conditions. It can be observed here that the red/green illumination causes an increasing of the trapped charge (holes and electrons) within the intrinsic layer of the bottom diode, being this elfect more intense under red light conditions.

Under blue light conditions, we note an increasing of only the trapped electrons within the intrinsic layer of the bottom diode. The photo-generation is localized within the top diode, the holeelectron pairs are thereby separated and only the electrons cross the internal junction toward the bottom diode, where they may lall trapped.

The simulated distribution of the trapped charge is in agreement with the experimental results and justifies the different capacitance values observed under different illumination conditions, addressing the explanation of the device functioning as a wavelength tuneable photo-capacitance controlled through the accumulation of trapped charge localized in the intrinsic layer of the bottom diode. This conclusion is somehow an improvement of our first impression when we assumed that the photocapacitance would be controlled by the trapped charge at the interna] recombination junction.

\section{Conclusions and future work}

We have presented experimental results about the capacitance of a a-Si:H/a-SiC:H pinpin photodiode. The capacitance has been found to vary depending on the illumination conditions. When illuminated by a pulsed light the device presents a frequency response that leave open the possibility of developing a light activated and wavelength controlled frequency filter. The behaviour of the device has been explained recurring to a numerical simulation, pointing out the fact that the photo-capacitance is controlled through the accumulation of trapped charge localized in the intrinsic layer of the bottom diode. Such a photo-capacitance adds to the standard junction diode capacitance and must be considered placed in parallel with it. 1ts value is directly related to the intensity of the incident light.

The superposition of a pulsed light on the optical bias produces an added modification of the trapped charge distribution. A full understanding of these modifications is still needed in order to explain and optimize the frequency response of the device under complex conditions of illumination. As a future work, to produce a complete picture, the frequency response should be correlated to light wavelength and intensity. Also, the simulations should take into account the dynamic of the defect stats filling during the transient condition of the pulsed light switching on/off.

\section{Acknowledgments}

This work was supported by FCT (CTS multiannual funding) through the PIDDAC Program funds and PTDC/EEA-ELC/120539/ 2010.

\section{References}

[1] M.G. Kuzyk, Polymer Fiber Optics, Materials Physics and Applications, Taylor and Francis Group, 2007.

[2] O. Ziemann, J. Krauser. P.E. Zanzow, W. Daum, POF Handbook, Optical Shor Range Transmission Systenrs, second ed, Springer, 2008.

[3] P. Louro, M. Vieira, M. Fernandes, J. Costa, M.A. Vieira, J. Caeiro, N. Neves, M. Earata, Physica Status Solid, C 7 (3-4) (2010) 1188-1191.

[4] M.A. Vieira. M, Vięira, M, Fernandes, A. Fantoni, P. Louro, M. Barala, MRS Proceedings 1153 (2009) A08-03. 
[5] A. Fantoni, P. L.ouro, M.A. Vieiru, T. Silva. M. Vieıra, Sensors and Actuators A

Physical 186 (2012) 143-197.
[6] M. Vlerra, A. Fantoni, M. Fernandes, P. Louro, G. Lavareda. C.N Carvalho, lournal of Nanuscience and Nanutechuology 9 (6) (2009) 4022-4027.
[7] R. Martius, A. Fantonı, M. Vięra, Jouma] of Non-Crystalline Solids 164-16i6 (1993) 671-674

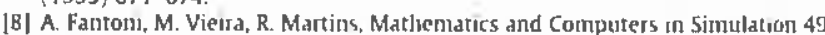
(1999) $381-401$. 